\title{
Infinite Horizon Optimal Policy for an Inventory System with Two Types of Products sharing Common Hardware Platforms
}

\author{
Mabel C. Chou, Chee-Khian Sim; Xue-Ming Yuan ${ }^{\ddagger}$
}

October 19, 2016

\begin{abstract}
We consider a periodic review inventory system and present its optimal policy in the infinite horizon setting. The optimal inventory policy that maximizes the infinite horizon expected discounted profit for the model is analytically obtained by relating to the finite horizon setting using results from variational analysis. Results are provided that elucidate the operations of the inventory system in the long run.
\end{abstract}

Keywords: Dynamic programming; inventory control; lateral transshipment; optimal policy; variational analysis.

\section{Introduction}

In this paper, we consider the problem of finding the infinite horizon optimal policy structure of an inventory system, and then analyze the behavior of the inventory system in the long run using the optimal policy structure that we obtained. The inventory system studied

*Department of Decision Sciences, NUS Business School, Mochtar Riady Building, BIZ 1 8-66, 15 Kent Ridge Drive, Singapore 119245. Email: bizchoum@nus.edu.sg

$\dagger$ Department of Mathematics, University of Portsmouth, Lion Gate Building, Lion Terrace, Portsmouth PO1 3HF. Email: chee-khian.sim@port.ac.uk (Corresponding author)

¥Singapore Institute of Manufacturing Technology, 2 Fusionopolis Way, \#08-04, Singapore 138634. Email: xmyuan@SIMTech.a-star.edu.sg 
in this paper is first discussed in Chou, Sim and Yuan [6]. In Chou, Sim and Yuan [6], this inventory system is studied when the planning horizon is finite. It is related to the well-known lateral transshipment problem. Our model of two types of product sharing a common hardware platform is similar to an inventory model with two locations, having different transshipment costs between them, and with demand faced by each location $\beta D$ and $(1-\beta) D$, respectively. Each hardware platform in our system can either be installed with software 1 or 2 in response to demand $\beta D$ for Product 1 and $(1-\beta) D$ for Product 2 , where $D$ is the total demand for the product, which has two types, Product 1 and Product 2. A product can be "converted" to the other product by uninstalling and then reinstalling softwares, after demand realization, using a policy similar to a type of transshipment policy which is called complete pooling (Tagaras [16]). We analytically derive the optimal inventory policy and hence the optimal proportion of an order to be installed with software 1 or 2 in the infinite horizon setting. In this paper, the stationary optimal policy for the inventory system comprising of two types of product sharing a common hardware platform is proven to be an order-up-to policy for each type of product. To understand the paper, we can either view the model as a "software-focused" model or a lateral transshipment model. We choose the former for ease in exposition. All results given can easily be translated into the language of a lateral transshipment problem.

Variational analysis has applications in the study of optimization, control, equilibrium problems, and stability of linear and nonlinear systems. In this paper, we provide another of its applications - dynamic programming in relation to inventory management. This, to the best of our knowledge, is something new in the inventory control literature. We use variational analysis results on limits (namely, Theorem 7.33 in Rockafellar and Wets [15]) to extend from the finite horizon scenario of our inventory model to its infinite horizon scenario. An existing approach (see, for example, Yuan and Cheung [20]) to extend from the finite horizon scenario to the infinite horizon scenario uses Theorems 8-14 and 8-15 in Heyman and Sobel [9]. We cannot apply these theorems in our study since these theorems, although applied in a general setting, assume that the action space lies on the real line. However, our inventory model has an action space that belongs to $\Re^{2}$. Theorem 7.33 in Rockafellar and Wets [15] is framed in a general Euclidean space setting $\left(\Re^{n}, n \geq 1\right)$, and hence it can readily be used to study our infinite horizon inventory model. A reference used in the inventory control literature to extend from the finite horizon scenario to the infinite horizon 
scenario is Section 1.2 of Bertsekas [3], where the one period cost function is assumed to be bounded. Unlike Theorem 7.33 in Rockafellar and Wets [15], we cannot use the results there easily for our model since our one period profit function is not bounded from below. Results in Chapter 4, in particular, Proposition 4.3.14, in Bertsekas [2] require monotonicity assumptions to be satisfied before they can be applied. These assumptions are not needed when we apply Theorem 7.33 in Rockafellar and Wets [15]. Feinberg [8] studies Markov decision processes and its application to periodic review inventory system with backorders, but the model we have in our study is a lost sales model.

An additional contribution of this paper, besides introducing a way to extend from the finite horizon scenario to the infinite horizon scenario, is the results we obtained that elucidate the operations of our inventory system in the long run and that appeal to our intuitive understanding. This is achieved by analyzing the structure of the infinite horizon optimal policy.

\subsection{Literature Review}

Relevant literature that puts particular attention on the study of periodic review inventory systems in the infinite horizon scenario includes the classical works by Iglehart [11], [12], Veinott [18], Zheng [21], and more recent works (after year 2000) Chen [4], Chen and SimchiLevi [5], Feng and Chen [7], van Ryzin and Vulcano [17], Yuan and Cheung [20]. These papers consider inventory models, with some considering joint inventory and pricing models, for either discounted or average profit/cost criteria or both. They consider a single product without transshipment, while in our paper, we consider two products that are allowed to be converted from one to the other. In Chen [4], the author performs steps similar to those outlined below in this paper to extend from the finite horizon setting to the infinite horizon setting, although no variational analysis results on limits are used. While in Chen and Simchi-Levi [5], the optimal policy in the infinite horizon setting is not obtained directly by a limiting procedure from the finite horizon optimal policy, and both policies do not generally have the same structure.

With regards to lateral transshipment, our study of the literature reveals that $\mathrm{Hu}$, Duenyas and Kapuscinski [10], and Yang and Qin [19] are two recent papers that study periodic review, infinite horizon transshipment model for discounted cost/profit by stochas- 
tic dynamic programming. Yang and Qin [19] provide detailed analysis on how they derive the optimal policy, while $\mathrm{Hu}$, Duenyas and Kapuscinski [10] consider the infinite horizon scenario as an extension to the main results in their paper. Also, we note that there is a brief mention in Ignall and Veinott [13] on how the finite horizon results in Ignall and Veinott [13] can be extended to infinite horizon.

Yang and Qin [19] study the transshipment problem for two capacitated manufacturing plants, with lateral transshipments being made virtually. A difference between their work and ours is that we consider a lost sales model, while in their model, demand can be backlogged. Also, the authors allow "virtual" transshipment whereby the inventory levels need not be nonnegative for transshipment to take place. This is not allowed in our model.

$\mathrm{Hu}$, Duenyas and Kapuscinski [10] study the optimal joint control of inventory and transshipment for a firm that produces in two locations and faces capacity uncertainty. They also consider a lost sales model, as in our case. An explicit optimal transshipment policy is given in $\mathrm{Hu}$, Duenyas and Kapuscinski [10]. However, the authors do not give an explicit formula for the order-up-to production policy. In this paper, we provide explicit formula for the optimal order-up-to policy in the infinite horizon setting.

For a detailed review on lateral transshipment models, the readers can refer to Paterson, et.al. [14]. A related paper to lateral transshipment is Agrawal, Chao and Seshadri [1].

We organize the paper as follows: In the next section, we describe our model and define notations that we use in the paper. In Section 3, we review the finite horizon optimal policy of our model and also state some new results. In Section 4, using results from Section 3, we obtain the infinite horizon optimal inventory policy for our model. Furthermore in the section, we analyze the optimal policy obtained and provide results on the operations of the inventory system in the long run. This paper is closely tied to Chou, Sim and Yuan [6], so we quote some definitions and results there in the paper.

\section{The Model}

We consider an inventory system with two types of product sharing a common hardware platform in a finite planning horizon $N$, and we let $N$ tend to infinity.

Parameters of the model are as follows: 
- $\sigma_{i}=$ installation cost to form a unit of Product $i, i=1,2$.

- $s_{i}=$ per unit selling price of Product $i, i=1,2$.

- $\tau_{1}=$ per unit cost for uninstalling the software for Product 2 and to install the software for Product 1, whenever there is shortage for Product 1 and excess of Product 2 for a given demand realization.

- $\tau_{2}=$ per unit cost for uninstalling Product 1 software and installing Product 2 software to form Product 2 when there is a shortage of Product 2 and excess of Product 1 for a given demand realization.

- $h=$ per unit time per unit inventory holding cost of hardware platform.

- $p=$ per unit unfilled penalty cost of hardware platform in a period.

- $c_{0}=$ per unit purchasing cost of hardware platform.

Randomness in the model comes from:

- $D=$ total demand for Products 1 and 2 in the $n^{\text {th }}$ period.

We assume that demands in different periods are independent and identically distributed.

- $\beta=$ proportion of $D$, with $\beta D$ the demand for Product 1 and $(1-\beta) D$ the demand for Product 2 in the $n^{\text {th }}$ period.

We have $0<\beta<1$. Note that $\beta$ is not dependent on $n$ and is a fixed number. This implies that demand for Product 1 and for Product 2 are perfectly correlated in a positive manner in each period. Perfect correlation of demands is the main restriction on our model which we hope to address in future.

Variables of the model are as follows:

- $x_{i, n}=$ inventory level of Product $i$ at the beginning of the $n^{\text {th }}$ period, $i=1,2$. Note that $x_{i, n} \geq 0, i=1,2$.

- $u_{i, n}=$ inventory level of Product $i$ after arrival of hardware platforms and installing these hardware platforms with different softwares in the $n^{\text {th }}$ period, $i=1,2$. Note that $u_{i, n} \geq x_{i, n}, i=1,2$. 
- $y_{n}=$ order quantity of common hardware platforms in the $n^{\text {th }}$ period.

We have $y_{n}=u_{1, n}+u_{2, n}-x_{1, n}-x_{2, n}$.

We assume that the lead time for common hardware platforms to arrive and the installation/uninstallation time of software are short enough to happen within the same period. The sequence of events for our model is such that at the beginning of the $n^{\text {th }}$ period, the initial inventory of Product $i$ is $x_{i, n}, i=1,2 . y_{n}$ units of hardware platform ordered, arrive immediately. These hardware platforms are then installed with either software 1 or 2 to make up a total of $u_{i, n}$ units of Product $i, i=1,2$, respectively. Next, demand is realized. Uninstalling of software and reinstalling of the other software occurs to satisfy shortage of the other product, whenever there is excess of the first product. Any demand for each product that is not satisfied is lost. Finally, different costs are calculated, and any excess of each product is brought over to the next period.

We have the following assumptions on parameters of our model throughout the paper:

Assumption 1 (a) $s_{i}>\sigma_{i}+c_{0}, i=1,2$.

(b) $\tau_{i}<h+p, i=1,2$.

(c) $s_{2}<s_{1}+\tau_{2}$ and $s_{1}<s_{2}+\tau_{1}$.

(d) $s_{1}-\sigma_{1} \geq s_{2}-\sigma_{2}$.

Assumption 1(a) is a standard assumption to avoid trivial consideration. Assumption 1(b) means that it is more advantageous to uninstall and reinstall softwares to get one unit of a type of product given a shortage of that type and excess of the other type. Assumption 1(c) ensures that if there is demand for Product 1 or Product 2, then any available stock of the product will be used to satisfy its own demand first before converting the available stock to the other product. Only excess stock is converted to satisfy any demand for the other product. Assumption 1(d) is a technical assumption that is made without loss of generality. It simply states that the profit margin of Product 1 is no lower than that of Product 2 .

The expected profit, $P_{n}$, in the $n^{\text {th }}$ period is given by:

$$
P_{n}\left(x_{1, n}, x_{2, n}, u_{1, n}, u_{2, n}\right)=\left(c_{0}+\sigma_{1}\right) x_{1, n}+\left(c_{0}+\sigma_{2}\right) x_{2, n}+\Phi\left(u_{1, n}, u_{2, n}\right),
$$


where

$$
\begin{aligned}
\Phi\left(u_{1}, u_{2}\right) \equiv & \left(s_{1}-c_{0}-\sigma_{1}\right) u_{1}+\left(s_{2}-c_{0}-\sigma_{2}\right) u_{2} \\
& -\left(s_{1}-\bar{s}_{2}\right) E\left(u_{1}-\beta D\right)^{+}-\left(s_{2}-\bar{s}_{1}\right) E\left(u_{2}-(1-\beta) D\right)^{+} \\
& -h E\left(u_{1}+u_{2}-D\right)^{+}-p E\left(D-u_{1}-u_{2}\right)^{+} \\
& -\bar{s}_{1} E\left(\left(u_{2}-(1-\beta) D\right)^{+}-\left(\beta D-u_{1}\right)^{+}\right)^{+} \\
& -\bar{s}_{2} E\left(\left(u_{1}-\beta D\right)^{+}-\left((1-\beta) D-u_{2}\right)^{+}\right)^{+}
\end{aligned}
$$

In $(2), \bar{s}_{i}$ is given by $s_{i}-\tau_{i}, i=1,2 . \bar{s}_{i}$ stands for the "effective" revenue in selling one unit of product $i$ that is converted from the other product after demand realization, $i=1,2$.

The expected total discounted profit from the $1^{\text {st }}$ period to the $N^{t h}$ period, for initial inventories $x_{1,1}, x_{2,1}$, is given by

$$
E\left[\sum_{n=1}^{N} \alpha^{n-1} P_{n}\left(x_{1, n}, x_{2, n}, u_{1, n}, u_{2, n}\right)\right],
$$

where for $n \geq 2$

$$
\left(x_{1, n}, x_{2, n}\right)=V\left(u_{1, n-1}-\beta D, u_{2, n-1}-(1-\beta) D\right),
$$

and $\alpha \in(0,1)$ is the discount factor.

Note that $V\left(v_{1}, v_{2}\right)$ is defined by

$$
V\left(v_{1}, v_{2}\right) \equiv\left(\left(v_{1}^{+}-v_{2}^{-}\right)^{+},\left(v_{2}^{+}-v_{1}^{-}\right)^{+}\right)
$$

$V\left(u_{1, n-1}-\beta D, u_{2, n-1}-(1-\beta) D\right)$ in $(4)$ gives us the physical units of Product $1, x_{1, n}$, and Product 2, $x_{2, n}$, available at the beginning of the $n^{\text {th }}$ period. Our model is such that whenever there is excess of one type of product and shortage of the other type after demand realization in the $(n-1)^{t h}$ period, all available units of that type is converted to satisfy the demand for the other type. This is similar to the complete pooling transshipment policy, as given in Tagaras [16]. Unsatisfied demand is lost.

In the infinite horizon expected discounted profit model, we determine the optimal policy that maximizes the infinite horizon expected discounted profit

$$
\liminf _{N \rightarrow \infty} E\left[\sum_{n=1}^{N} \alpha^{n-1} P_{n}\left(x_{1, n}, x_{2, n}, u_{1, n}, u_{2, n}\right)\right],
$$

for $0<\alpha<1$, and any nonnegative initial inventory levels $x_{1,1}, x_{2,1}$, through finding the optimal $u_{1, n}$ and $u_{2, n}$. Here a feasible policy is understood to be a given $\left(u_{1, n}, u_{2, n}\right)$ with 
$u_{1, n} \geq x_{1, n}, u_{2, n} \geq x_{2, n}$ for $n \geq 1$. We show that the optimal policy is stationary in that the optimal $u_{1, n}$ and $u_{2, n}$ are given by expressions that depend on $n$ only through $x_{1, n}$ and $x_{2, n}$. The optimal policy is observed to have an "order-up-to" structure.

To extend from the finite horizon scenario to the infinite horizon scenario, we follow the below steps, which provide a systematic way which may be useful in rigorously establishing infinite horizon optimal policies of other inventory models from their finite horizon counterparts:

1. Use standard analysis to show that the optimal finite horizon expected discounted profit from period 1 to period $N$ and its derivatives converge (uniformly) to a function and its derivatives, as $N \rightarrow \infty$.

2. Use variational analysis results on limits to show that the function satisfies a Bellmantype equation and that the optimal $u_{1,1}, u_{2,1}$ for the profit from period 1 to period $N$ converge to the optimal solution in the Bellman-type equation, as $N \rightarrow \infty$. The structure of the optimal solution, which becomes the structure of the infinite horizon optimal policy, is obtained separately for our inventory model (Theorem 2).

3. Use the Bellman-type equation to show that the optimal solution in the Bellman-type equation is a feasible infinite horizon policy with the function being the corresponding infinite horizon expected discounted profit function. Use these to conclude that the function is in fact the optimal infinite horizon expected discounted profit function with the optimal solution being the infinite horizon optimal policy.

\section{Finite Horizon: Review and New Results}

To find an optimal policy that maximizes (3), for $1 \leq n \leq N$, let $\pi_{n}^{N}\left(x_{1, n}, x_{2, n}\right)$ be the optimal discounted profit from the $n^{\text {th }}$ period to the $N^{\text {th }}$ period in the $N$ period planning horizon, given that the inventory levels of Products 1 and 2 at the beginning of the $n^{\text {th }}$ period are $x_{1, n}(\geq 0)$ and $x_{2, n}(\geq 0)$, respectively. We have in dynamic programming formulation the following relation relating $\pi_{n}^{N}$ to each other:

$$
\begin{aligned}
& \pi_{n}^{N}\left(x_{1, n}, x_{2, n}\right)=\left(c_{0}+\sigma_{1}\right) x_{1, n}+\left(c_{0}+\sigma_{2}\right) x_{2, n} \\
& +\max _{u_{1} \geq x_{1, n}, u_{2} \geq x_{2, n}}\left\{\Phi\left(u_{1}, u_{2}\right)+\alpha E \pi_{n+1}^{N}\left(V\left(u_{1}-\beta D, u_{2}-(1-\beta) D\right)\right)\right\},
\end{aligned}
$$


where we define $\pi_{N+1}^{N}\left(x_{1, N+1}, x_{2, N+1}\right) \equiv 0$ for all $x_{1, N+1}(\geq 0)$ and $x_{2, N+1}(\geq 0)$.

Let

$$
\Psi_{n}^{N}\left(u_{1}, u_{2}\right) \equiv \Phi\left(u_{1}, u_{2}\right)+\alpha E \pi_{n+1}^{N}\left(V\left(u_{1}-\beta D, u_{2}-(1-\beta) D\right)\right) .
$$

Hence,

$$
\pi_{n}^{N}\left(x_{1, n}, x_{2, n}\right)=\left(c_{0}+\sigma_{1}\right) x_{1, n}+\left(c_{0}+\sigma_{2}\right) x_{2, n}+\max _{u_{1} \geq x_{1, n}, u_{2} \geq x_{2, n}} \Psi_{n}^{N}\left(u_{1}, u_{2}\right) .
$$

We have in the following theorem, an optimal policy that maximizes (3), by solving (5):

Theorem 1 [Theorem 4, Chou, Sim and Yuan [6]] For $1 \leq n \leq N$ and $x_{1, n}, x_{2, n} \geq 0$, the optimal solution $\left(u_{1, n}^{N, *}, u_{2, n}^{N, *}\right)$ to

$$
\pi_{n}^{N}\left(x_{1, n}, x_{2, n}\right)=\left(c_{0}+\sigma_{1}\right) x_{1, n}+\left(c_{0}+\sigma_{2}\right) x_{2, n}+\max _{u_{1} \geq x_{1, n}, u_{2} \geq x_{2, n}} \Psi_{n}^{N}\left(u_{1}, u_{2}\right)
$$

is given by

$$
u_{1, n}^{N, *}=\max \left\{x_{1, n}, \min \left\{\bar{u}_{1, n}^{N}\left(x_{2, n}\right), \bar{u}_{1, n}^{N, *}\right\}\right\}, u_{2, n}^{N, *}=\max \left\{x_{2, n}, \min \left\{\bar{u}_{2, n}^{N}\left(x_{1, n}\right), \bar{u}_{2, n}^{N, *}\right\}\right\}
$$

where $\left(\bar{u}_{1, n}^{N, *}, \bar{u}_{2, n}^{N, *}\right)$ solves the following system of equations:

$$
\left.\begin{array}{rl}
\frac{\partial \Psi_{n}^{N}}{\partial u_{1}}\left(u_{1}, u_{2}\right) & =0 \\
\frac{\partial \Psi_{n}^{N}}{\partial u_{2}}\left(u_{1}, u_{2}\right) & =0
\end{array}\right\}
$$

for $u_{1}, u_{2} \geq 0$.

$\bar{u}_{1, n}^{N}\left(x_{2, n}\right)$ satisfies $\frac{\partial \Psi_{n}^{N}}{\partial u_{1}}\left(\bar{u}_{1, n}^{N}\left(x_{2, n}\right), x_{2, n}\right)=0$, and $\bar{u}_{2, n}^{N}\left(x_{1, n}\right)$ satisfies $\frac{\partial \Psi_{n}^{N}}{\partial u_{2}}\left(x_{1, n}, \bar{u}_{2, n}^{N}\left(x_{1, n}\right)\right)=0$.

We adopt the convention that if there are no solutions to the above equation system, then $\bar{u}_{1, n}^{N, *}=\infty$ and $\bar{u}_{2, n}^{N, *}=-\infty$.

We know from Chou, Sim and Yuan [6] that $\pi_{1}^{N}\left(x_{1}, x_{2}\right)$ is a concave, continuously differentiable function of $\left(x_{1}, x_{2}\right), x_{1}, x_{2} \geq 0$, for all $N \geq 1$. In Propositions 2 and 3 below, which are new, we investigate how $\pi_{1}^{N}$ and its first order partial derivatives behave as $N \rightarrow \infty$. Before stating these new propositions, we introduce $W\left(v_{1}, v_{2}\right) \equiv\left(v_{1}^{+}-v_{2}^{-}\right)^{+}$. This function, which is related to $V\left(v_{1}, v_{2}\right)$ defined in Section 2 by $V\left(v_{1}, v_{2}\right)=\left(W\left(v_{1}, v_{2}\right), W\left(v_{2}, v_{1}\right)\right)$, and its useful properties, as stated in Proposition 1, are needed in the proof of Propositions 2 and 3 . 
Proposition 1 [Proposition 8, Chou, Sim and Yuan [6]] (i) As v $v_{1}$ or $v_{2}$ increases (decreases), both $W\left(v_{1}, v_{2}\right)$ and $W\left(v_{2}, v_{1}\right)$ are nondecreasing (nonincreasing). If, as both $v_{1}$ and $v_{2}$ are increasing, $W\left(v_{1}, v_{2}\right)$ or $W\left(v_{2}, v_{1}\right)$ is a constant, then the constant is zero.

(ii) For $v_{1} \neq 0, v_{2} \neq 0$ and $v_{1}+v_{2} \neq 0$, we have $W\left(v_{1}, v_{2}\right)$ is twice continuously differentiable with

$$
\begin{aligned}
& \frac{\partial W}{\partial v_{1}}\left(v_{1}, v_{2}\right)=\left\{\begin{array}{ll}
1 & , \text { if } v_{1}>v_{2}^{-} \\
0 & , \text { otherwise }
\end{array},\right. \\
& \frac{\partial W}{\partial v_{2}}\left(v_{1}, v_{2}\right)=\left\{\begin{array}{ll}
1 & , \text { if } v_{1}+v_{2}>0, v_{1}>0, v_{2}<0 \\
0 & , \text { otherwise }
\end{array},\right.
\end{aligned}
$$

and the second order partial derivatives of $W$ are equal to zero.

We have

Proposition $2 \frac{\partial \pi_{1}^{N}}{\partial x_{i}}\left(x_{1}, x_{2}\right)$ converges uniformly on $\Re_{+}^{2}$, as $N \rightarrow \infty$, for $i=1,2$.

Proof: To prove the proposition, we show that $\frac{\partial \pi_{1}^{N}}{\partial x_{i}}\left(x_{1}, x_{2}\right)=\sum_{k=1}^{N} C_{k, i}^{N}\left(x_{1}, x_{2}\right) \alpha^{k-1}, N \geq 1$, where $\left|C_{k, i}^{N}\left(x_{1}, x_{2}\right)\right| \leq C, 1 \leq k \leq N, i=1,2$, on $\Re_{+}^{2}$ by induction on $N$. Here, $C$ is some constant independent of $N$. We do this by using explicit expressions for $\frac{\partial \pi_{1}^{N}}{\partial x_{i}}\left(x_{1}, x_{2}\right), i=1,2$, that can be found in the proof of Proposition 6 in Chou, Sim and Yuan [6].

Let $C$ be an upper bound to $c_{0}+\sigma_{1}+\left|\frac{\partial \Phi}{\partial u_{1}}\left(x_{1}, x_{2}\right)\right|, c_{0}+\sigma_{1}+\mid \frac{\partial \Phi}{\partial u_{1}}\left(x_{1}, \bar{u}_{2,1}^{N}\left(x_{1}\right) \mid, c_{0}+\sigma_{2}+\right.$ $\left|\frac{\partial \Phi}{\partial u_{2}}\left(x_{1}, x_{2}\right)\right|, c_{0}+\sigma_{2}+\left|\frac{\partial \Phi}{\partial u_{2}}\left(\bar{u}_{1,1}^{N}\left(x_{2}\right), x_{2}\right)\right|$ on $\Re_{+}^{2}$, for all $N \geq 1$.

Such a $C$ exists as $\frac{\partial \Phi}{\partial u_{1}}\left(x_{1}, x_{2}\right)$ and $\frac{\partial \Phi}{\partial u_{2}}\left(x_{1}, x_{2}\right)$ are bounded by a constant for all $\left(x_{1}, x_{2}\right) \in \Re_{+}^{2}$. Hence, for $N=1$, the statement is true.

Suppose statement is true for $N=N_{0}$, that is, $\frac{\partial \pi_{1}^{N_{0}}}{\partial x_{i}}\left(x_{1}, x_{2}\right)=\sum_{k=1}^{N_{0}} C_{k, i}^{N_{0}}\left(x_{1}, x_{2}\right) \alpha^{k-1}$, where $\left|C_{k, i}^{N_{0}}\left(x_{1}, x_{2}\right)\right| \leq C, 1 \leq k \leq N_{0}, i=1,2$, on $\Re_{+}^{2}$.

Using expressions for $\frac{\partial \pi_{1}^{N_{0}+1}}{\partial x_{i}}\left(x_{1}, x_{2}\right), i=1,2$, as found in Chou, Sim and Yuan [6], which are expressed in terms of first order partial derivatives of $\pi_{2}^{N_{0}+1}$, it follows by induction hypothesis that $\frac{\partial \pi_{1}^{N_{0}+1}}{\partial x_{i}}\left(x_{1}, x_{2}\right)=\sum_{k=1}^{N_{0}+1} C_{k, i}^{N_{0}+1}\left(x_{1}, x_{2}\right) \alpha^{k-1}$, where $\left|C_{k, i}^{N_{0}+1}\left(x_{1}, x_{2}\right)\right| \leq C, 1 \leq k \leq N_{0}+1$, $i=1,2$, on $\Re_{+}^{2}$. This is possible by making the crucial observation that if there are two terms within expectation in these expressions, both terms cannot be nonzero at the same time due to properties of first order partial derivatives of $W$ as given in Proposition 1(ii). Hence, statement is true for $N=N_{0}+1$. Therefore, statement is true for all $N \geq 1$ by induction. 
Since, $\frac{\partial \pi_{1}^{N}}{\partial x_{i}}\left(x_{1}, x_{2}\right), i=1,2$, is an $N^{t h}$ partial sum in $\alpha$ bounded by the $N^{t h}$ partial sum of a convergence power series in $\alpha$, the proposition is proved.

The idea behind the proof of the above proposition is to write each first order partial derivative as an $N^{t h}$ partial sum of $\alpha$ which is bounded uniformly above on $\Re_{+}^{2}$ by the $N^{t h}$ partial sum of a convergent power series in $\alpha$. The result then follows by applying standard results from analysis.

Similarly, we have

Proposition $3 \pi_{1}^{N}\left(x_{1}, x_{2}\right)$ converges uniformly on bounded sets in $\Re_{+}^{2}$, as $N \rightarrow \infty$.

Since the proof of Proposition 3 is along the same line as that of Proposition 2, it is omitted. Propositions 2 and 3 give rise to the following immediately:

Proposition $4 \pi_{1}^{N}\left(x_{1}, x_{2}\right)$ converges uniformly to a continuously differentiable function $\pi_{\infty}\left(x_{1}, x_{2}\right)$ on bounded sets in $\Re_{+}^{2}$, with its first order partial derivatives converging uniformly to the first order partial derivatives of $\pi_{\infty}\left(x_{1}, x_{2}\right)$ on $\Re_{+}^{2}$.

Remark 1 Proposition 4, using Lemma 1 of Chou, Sim and Yuan [6], tells us that $\alpha E\left(\pi_{\infty}\left(V\left(u_{1}-\right.\right.\right.$ $\left.\left.\left.\beta D, u_{2}-(1-\beta) D\right)\right)\right)$ is continuously differentiable for all $\left(u_{1}, u_{2}\right), u_{1}, u_{2}, \geq 0$. Also, $\alpha E\left(\pi_{1}^{N}\left(V\left(u_{1}-\right.\right.\right.$ $\left.\left.\left.\beta D, u_{2}-(1-\beta) D\right)\right)\right)$ converges uniformly to $\alpha E\left(\pi_{\infty}\left(V\left(u_{1}-\beta D, u_{2}-(1-\beta) D\right)\right)\right)$ on bounded sets in $\Re_{+}^{2}$, with its first order partial derivatives converging uniformly to that of $\alpha E\left(\pi_{\infty}\left(V\left(u_{1}-\beta D, u_{2}-(1-\beta) D\right)\right)\right)$ on $\Re_{+}^{2}$, as $N \rightarrow \infty$.

Note that $\pi_{\infty}\left(x_{1}, x_{2}\right)$ is the candidate for the optimal infinite horizon expected discounted profit, as we will show in the next section.

\section{Infinite Horizon}

In this section, we derive the optimal policy for our model in the infinite horizon setting.

The following theorem tells us the structure which the stationary infinite horizon optimal policy takes:

Theorem 2 If $K_{\infty}\left(u_{1}, u_{2}\right)$ is such that

(a) $K_{\infty}\left(u_{1}, u_{2}\right)$ is a continuously differentiable, concave function of $\left(u_{1}, u_{2}\right), u_{1}, u_{2} \geq 0$, 
(b) $\frac{\partial K_{\infty}}{\partial u_{1}}(0,0)>c_{0}+\sigma_{1}-s_{1}-p, \lim _{u_{1} \rightarrow \infty} \frac{\partial K_{\infty}}{\partial u_{1}}\left(u_{1}, u_{2}\right)<c_{0}+\sigma_{1}+h$ for fixed $u_{2} \geq 0$,

(c) $\frac{\partial K_{\infty}}{\partial u_{2}}(0,0)>c_{0}+\sigma_{2}-s_{2}-p, \lim _{u_{2} \rightarrow \infty} \frac{\partial K_{\infty}}{\partial u_{2}}\left(u_{1}, u_{2}\right)<c_{0}+\sigma_{2}+h$ for fixed $u_{1} \geq 0$,

(d) $\frac{\partial K_{\infty}}{\partial u_{1}}\left(u_{1}, u_{2}\right)$ is nonincreasing in $u_{2}$ for fixed $u_{1} \geq 0$, and $\frac{\partial K_{\infty}}{\partial u_{2}}\left(u_{1}, u_{2}\right)$ is nonincreasing in $u_{1}$ for fixed $u_{2} \geq 0$,

(e) $\frac{\partial K_{\infty}}{\partial u_{1}}\left(0, u_{2}\right)=\frac{\partial K_{\infty}}{\partial u_{2}}\left(0, u_{2}\right)$ for all $u_{2} \geq 0$,

(f) $\frac{\partial K_{\infty}}{\partial u_{1}}\left(u_{1}, 0\right)=\frac{\partial K_{\infty}}{\partial u_{2}}\left(u_{1}, 0\right)$ for all $u_{1} \geq 0$,

then for $x_{1}, x_{2} \geq 0$,

$$
\left(c_{0}+\sigma_{1}\right) x_{1}+\left(c_{0}+\sigma_{2}\right) x_{2}+\max _{u_{1} \geq x_{1}, u_{2} \geq x_{2}} \Psi_{\infty}\left(u_{1}, u_{2}\right),
$$

where $\Psi_{\infty}\left(u_{1}, u_{2}\right) \equiv \Phi\left(u_{1}, u_{2}\right)+K_{\infty}\left(u_{1}, u_{2}\right)$, has an unique optimal solution $\left(u_{1, \infty}^{*}, u_{2, \infty}^{*}\right)$ given by

$$
u_{1, \infty}^{*}=\max \left\{x_{1}, \min \left\{\bar{u}_{1, \infty}\left(x_{2}\right), \bar{u}_{1, \infty}^{*}\right\}\right\}, u_{2, \infty}^{*}=\max \left\{x_{2}, \min \left\{\bar{u}_{2, \infty}\left(x_{1}\right), \bar{u}_{2, \infty}^{*}\right\}\right\},
$$

where $\left(\bar{u}_{1, \infty}^{*}, \bar{u}_{2, \infty}^{*}\right)$ solves the following system of equations:

$$
\left.\begin{array}{l}
\frac{\partial \Psi_{\infty}}{\partial u_{1}}\left(u_{1}, u_{2}\right)=0 \\
\frac{\partial \Psi_{\infty}}{\partial u_{2}}\left(u_{1}, u_{2}\right)=0
\end{array}\right\}
$$

for $u_{1}, u_{2} \geq 0$.

$\bar{u}_{1, \infty}\left(x_{2}\right)$ satisfies $\frac{\partial \Psi_{\infty}}{\partial u_{1}}\left(\bar{u}_{1, \infty}\left(x_{2}\right), x_{2}\right)=0$, and $\bar{u}_{2, \infty}\left(x_{1}\right)$ satisfies $\frac{\partial \Psi_{\infty}}{\partial u_{2}}\left(x_{1}, \bar{u}_{2, \infty}\left(x_{1}\right)\right)=0$.

We adopt the convention that if there are no solutions to the above equation system, then $\bar{u}_{1, \infty}^{*}=\infty$ and $\bar{u}_{2, \infty}^{*}=-\infty$.

Proof: The results in this theorem are analogous to that in Theorem 1 in Chou, Sim and Yuan [6], which holds under Conditions 1-6 in the paper. Compared to these conditions, we have in this theorem, analogous conditions, other than the condition given in (d) in place of Condition 4. It can be checked easily that the results in Chou, Sim and Yuan [6] leading up to Theorem 1 in the paper holds under the weaker condition given in (d), together with the other conditions. Hence, following the arguments in Chou, Sim and Yuan [6], this theorem is proved. 
Remark 2 We note that in Theorem 2 above, we have given a new weaker condition, namely, condition given in (d), together with the other conditions, for Theorem 1 in Chou, Sim and Yuan [6] to hold.

The conditions in Theorem 2 are satisfied by $\alpha E \pi_{\infty}\left(V\left(u_{1}-\beta D, u_{2}-(1-\beta) D\right)\right)$, as shown in the following proposition:

Proposition $5 \alpha E \pi_{\infty}\left(V\left(u_{1}-\beta D, u_{2}-(1-\beta) D\right)\right)$ satisfies the conditions in Theorem 2.

Proof: Follows from Remark 1 and that these conditions hold for $\alpha E \pi_{1}^{N}\left(V\left(u_{1}-\beta D, u_{2}-\right.\right.$ $(1-\beta) D)), \forall N \geq 1$ (Proposition 9 and its proof in Chou, Sim and Yuan [6]).

Proposition 5 shows that the conditions on $K_{\infty}\left(u_{1}, u_{2}\right)$ in Theorem 2 are satisfied by $\alpha E \pi_{\infty}\left(V\left(u_{1}-\beta D, u_{2}-(1-\beta) D\right)\right)$. Hence, the results in the theorem can be applied to our model.

Note that so far, we have not shown that the optimal solution given in Theorem 2 forms the infinite horizon optimal policy. In the following, using variational analysis results on limits (Theorem 7.33 in Rockafellar and Wets [15]), we are able to show that $\pi_{\infty}$ satisfies a Bellman-type equation, and also relate the optimal solution of $\pi_{1}^{N}$ to that in Theorem 2 . This is crucial to allow us to conclude that $\pi_{\infty}$ is indeed the optimal infinite horizon expected discounted profit and the optimal solution in Theorem 2 forms the infinite horizon optimal policy.

Theorem 3 For $x_{1}, x_{2} \geq 0, \pi_{\infty}\left(x_{1}, x_{2}\right)$ satisfies

$$
\pi_{\infty}\left(x_{1}, x_{2}\right)=\left(c_{0}+\sigma_{1}\right) x_{1}+\left(c_{0}+\sigma_{2}\right) x_{2}+\max _{u_{1} \geq x_{1}, u_{2} \geq x_{2}} \Psi_{\infty}\left(u_{1}, u_{2}\right)
$$

where

$$
\Psi_{\infty}\left(u_{1}, u_{2}\right)=\Phi\left(u_{1}, u_{2}\right)+\alpha E \pi_{\infty}\left(V\left(u_{1}-\beta D, u_{2}-(1-\beta) D\right)\right) .
$$

The unique optimal solution $\left(u_{1, \infty}^{*}, u_{2, \infty}^{*}\right)$ to (7) is given by

$$
u_{1, \infty}^{*}=\max \left\{x_{1}, \min \left\{\bar{u}_{1, \infty}\left(x_{2}\right), \bar{u}_{1, \infty}^{*}\right\}\right\}, u_{2, \infty}^{*}=\max \left\{x_{2}, \min \left\{\bar{u}_{2, \infty}\left(x_{1}\right), \bar{u}_{2, \infty}^{*}\right\}\right\},
$$

where $\left(\bar{u}_{1, \infty}^{*}, \bar{u}_{2, \infty}^{*}\right)$ solves the following system of equations:

$$
\left.\begin{array}{l}
\frac{\partial \Psi_{\infty}}{\partial u_{1}}\left(u_{1}, u_{2}\right)=0 \\
\frac{\partial \Psi_{\infty}}{\partial u_{2}}\left(u_{1}, u_{2}\right)=0
\end{array}\right\}
$$


for $u_{1}, u_{2} \geq 0$.

$\bar{u}_{1, \infty}\left(x_{2}\right)$ satisfies $\frac{\partial \Psi_{\infty}}{\partial u_{1}}\left(\bar{u}_{1, \infty}\left(x_{2}\right), x_{2}\right)=0$, and $\bar{u}_{2, \infty}\left(x_{1}\right)$ satisfies $\frac{\partial \Psi_{\infty}}{\partial u_{2}}\left(x_{1}, \bar{u}_{2, \infty}\left(x_{1}\right)\right)=0$.

We adopt the convention that if there are no solutions to the above equation system, then $\bar{u}_{1, \infty}^{*}=\infty$ and $\bar{u}_{2, \infty}^{*}=-\infty$.

Also, $\left(u_{1,1}^{N, *}, u_{2,1}^{N, *}\right) \rightarrow\left(u_{1, \infty}^{*}, u_{2, \infty}^{*}\right)$ as $N \rightarrow \infty$, with $\left(u_{1,1}^{N, *}, u_{2,1}^{N, *}\right)$ being the optimal solution to (6), where $n=1$.

Proof: By Theorem 2 using Proposition 5, we immediately have that

$$
\left(c_{0}+\sigma_{1}\right) x_{1}+\left(c_{0}+\sigma_{2}\right) x_{2}+\max _{u_{1} \geq x_{1}, u_{2} \geq x_{2}} \Psi_{\infty}\left(u_{1}, u_{2}\right),
$$

where $\Psi_{\infty}\left(u_{1}, u_{2}\right)=\Phi\left(u_{1}, u_{2}\right)+\alpha E \pi_{\infty}\left(V\left(u_{1}-\beta D, u_{2}-(1-\beta) D\right)\right)$, has unique optimal solution $\left(u_{1, \infty}^{*}, u_{2, \infty}^{*}\right)$ with properties given in the theorem statement.

We now show that the expression in (8) is actually equal to $\pi_{\infty}\left(x_{1}, x_{2}\right)$, hence showing that $\pi_{\infty}$ satisfies a Bellman-type equation. We use Theorem 7.33 in Rockafellar and Wets [15] to do this. To apply the theorem to our case, its assumptions on epi-convergence of $\Psi_{1}^{N}\left(u_{1}, u_{2}\right)$ to $\Psi_{\infty}\left(u_{1}, u_{2}\right)$, and eventually level-boundedness of $\left\{\Psi_{1}^{N}\left(u_{1}, u_{2}\right)\right\}$ need to be satisfied. These hold true if Theorem 7.17 and Exercise 7.32(c) in Rockafellar and Wets [15] respectively are true in our case.

Remark 1, which follows from Proposition 4, together with concavity of the functions involved, implies Theorem 7.17. Also, $\Psi_{\infty}\left(u_{1}, u_{2}\right)$ is level-bounded (which means that $\left\{\left(u_{1}, u_{2}\right) ; \Psi_{\infty}\left(u_{1}, u_{2}\right) \geq c\right\}$ is bounded for all $c$, and which follows since $\Psi_{\infty}\left(u_{1}, u_{2}\right)$ is concave with unique optimal solution), with $\Psi_{1}^{N}\left(u_{1}, u_{2}\right)$ concave for all $N \geq 1$, implies Exercise 7.32(c). Hence, we can apply Theorem 7.33 in Rockafellar and Wets [15] to our case, and by the theorem, we have

$$
\pi_{1}^{N}\left(x_{1}, x_{2}\right)=\left(c_{0}+\sigma_{1}\right) x_{1}+\left(c_{0}+\sigma_{2}\right) x_{2}+\max _{u_{1} \geq x_{1}, u_{2} \geq x_{2}} \Psi_{1}^{N}\left(u_{1}, u_{2}\right)
$$

converges to expression (8), with

$$
\left(u_{1,1}^{N, *}, u_{2,1}^{N, *}\right) \rightarrow\left(u_{1, \infty}^{*}, u_{2, \infty}^{*}\right), \quad \text { as } N \rightarrow \infty
$$

On the other hand, by Proposition $4, \pi_{1}^{N}\left(x_{1}, x_{2}\right) \rightarrow \pi_{\infty}\left(x_{1}, x_{2}\right)$ as $N \rightarrow \infty, x_{1}, x_{2} \geq 0$. Putting everything together, above shows that $\pi_{\infty}\left(x_{1}, x_{2}\right)$ satisfies

$$
\pi_{\infty}\left(x_{1}, x_{2}\right)=\left(c_{0}+\sigma_{1}\right) x_{1}+\left(c_{0}+\sigma_{2}\right) x_{2}+\max _{u_{1} \geq x_{1}, u_{2} \geq x_{2}} \Psi_{\infty}\left(u_{1}, u_{2}\right)
$$


where

$$
\Psi_{\infty}\left(u_{1}, u_{2}\right)=\Phi\left(u_{1}, u_{2}\right)+\alpha E \pi_{\infty}\left(V\left(u_{1}-\beta D, u_{2}-(1-\beta) D\right)\right) .
$$

Finally, using Theorem 3, in the following theorem, we obtain the stationary optimal policy for our infinite horizon expected discounted profit model:

Theorem 4 The optimal policy that maximizes

$$
\liminf _{N \rightarrow \infty} E\left[\sum_{n=1}^{N} \alpha^{n-1} P_{n}\left(x_{1, n}, x_{2, n}, u_{1, n}, u_{2, n}\right)\right]
$$

for $0<\alpha<1$, and any nonnegative initial inventory levels $x_{1,1}, x_{2,1}$, is given by

$$
u_{1, \infty}^{*}=\max \left\{x_{1, n}, \min \left\{\bar{u}_{1, \infty}\left(x_{2, n}\right), \bar{u}_{1, \infty}^{*}\right\}\right\}, u_{2, \infty}^{*}=\max \left\{x_{2, n}, \min \left\{\bar{u}_{2, \infty}\left(x_{1, n}\right), \bar{u}_{2, \infty}^{*}\right\}\right\},
$$

for all $n \geq 1$.

The optimal infinite horizon expected discounted profit function is given by $\pi_{\infty}\left(x_{1,1}, x_{2,1}\right)$.

Proof: It is clear that for each $N \geq 1$,

$$
E\left[\sum_{n=1}^{N} \alpha^{n-1} P_{n}\left(x_{1, n}, x_{2, n}, u_{1, n}, u_{2, n}\right)\right] \leq \pi_{1}^{N}\left(x_{1,1}, x_{2,1}\right) .
$$

Hence, given any infinite horizon feasible policy for the system, its expected discounted profit $\liminf _{N \rightarrow \infty} E\left[\sum_{n=1}^{N} \alpha^{n-1} P_{n}\left(x_{1, n}, x_{2, n}, u_{1, n}, u_{2, n}\right)\right]$ must satisfy

$$
\liminf _{N \rightarrow \infty} E\left[\sum_{n=1}^{N} \alpha^{n-1} P_{n}\left(x_{1, n}, x_{2, n}, u_{1, n}, u_{2, n}\right)\right] \leq \liminf _{N \rightarrow \infty} \pi_{1}^{N}\left(x_{1,1}, x_{2,1}\right)=\pi_{\infty}\left(x_{1,1}, x_{2,1}\right) .
$$

On the other hand,

$$
u_{1, \infty}^{*}=\max \left\{x_{1, n}, \min \left\{\bar{u}_{1, \infty}\left(x_{2, n}\right), \bar{u}_{1, \infty}^{*}\right\}\right\}, u_{2, \infty}^{*}=\max \left\{x_{2, n}, \min \left\{\bar{u}_{2, \infty}\left(x_{1, n}\right), \bar{u}_{2, \infty}^{*}\right\}\right\},
$$

for all $n \geq 1$, is a feasible policy with its infinite horizon expected discounted profit given by

$$
\liminf _{N \rightarrow \infty} E\left[\sum_{n=1}^{N} \alpha^{n-1} P_{n}\left(x_{1, n}, x_{2, n}, u_{1, \infty}^{*}, u_{2, \infty}^{*}\right)\right]=\pi_{\infty}\left(x_{1,1}, x_{2,1}\right)
$$

where the above equality holds since, by Theorem $3, \pi_{\infty}\left(x_{1}, x_{2}\right)$ satisfies $(7)$, with $\left(u_{1, \infty}^{*}, u_{2, \infty}^{*}\right)$ being the optimal solution to the maximization problem in (7).

Hence, the theorem is proved. 
Given an initial inventory of Product $1, x_{1}(\geq 0)$, and Product $2, x_{2}(\geq 0)$. If there are many units of Product 1 compared to the number of units of Product 2, namely, when $x_{1}$ exceeds $\min \left\{\bar{u}_{1, \infty}\left(x_{2}\right), \bar{u}_{1, \infty}^{*}\right\}$, then under optimality, we do not produce any more of Product 1. If there are not many units of Product 1 initially, then more units of Product 1 need to be produced to satisfy its demand. In this case, the number of units of Product 1 to produce depends on $x_{2}$, the initial inventory level of Product 2 . If $x_{2}$ is small, namely $x_{2} \leq \bar{u}_{2, \infty}^{*}$, then produce up to $\bar{u}_{1, \infty}^{*}$ units of Product 1. If the number of units of Product 2 exceeds $\bar{u}_{2, \infty}^{*}$, then produce less of Product 1, since Product 2 can be used to cover shortage of Product 1. How much less depends on $x_{2}$ - to be precise, in this case, produce only up to $\bar{u}_{1, \infty}\left(x_{2}\right)$ of Product 1, just enough, so that the marginal increase in expected profit in producing an extra unit of Product 1, given $x_{2}$, is zero. Similar argument holds for how many units of Product 2 to produce optimally, given $x_{1}, x_{2}$.

For $n \geq 1$, given realization $D_{n}=d_{n}$ of demand, which we assume to be larger than some constant (say 1), we now investigate the operations of the inventory system for $n$ large, when the planning horizon is infinite. We have a result given in the following theorem:

Theorem 5 Assume that $\bar{u}_{i, \infty}^{*}, i=1,2$, are finite. For any nonnegative initial inventory levels $x_{1,1}, x_{2,1}$, the optimal policy is such that for $n$ large enough, the inventory level of Product $i$ after arrival of hardware platforms and installing these hardware platforms with different softwares in the $n^{\text {th }}$ period, before demand realization, is always given by $\bar{u}_{i, \infty}^{*}$, $i=1,2$.

Proof: From Theorem 4, we know that for any nonnegative initial inventory levels $x_{1,1}, x_{2,1}$, the optimal policy is given by

$$
u_{1, \infty}^{*}=\max \left\{x_{1, n}, \min \left\{\bar{u}_{1, \infty}\left(x_{2, n}\right), \bar{u}_{1, \infty}^{*}\right\}\right\}, u_{2, \infty}^{*}=\max \left\{x_{2, n}, \min \left\{\bar{u}_{2, \infty}\left(x_{1, n}\right), \bar{u}_{2, \infty}^{*}\right\}\right\},
$$

for $n \geq 1$.

To prove the theorem, we only need to show that for all $n$ large enough, we have $u_{1, \infty}^{*}=$ $\bar{u}_{1, \infty}^{*}, u_{2, \infty}^{*}=\bar{u}_{2, \infty}^{*}$.

These hold true if $x_{1, n} \leq \bar{u}_{1, \infty}^{*}$ and $x_{2, n} \leq \bar{u}_{2, \infty}^{*}$ for all $n$ large, which we show below.

Note that by (4), we have for $n \geq 1$,

$$
x_{1, n} \leq\left(u_{1, \infty}^{*}-\beta d_{n-1}\right)^{+}, x_{2, n} \leq\left(u_{2, \infty}^{*}-(1-\beta) d_{n-1}\right)^{+} .
$$


Therefore, we either have $x_{1, n} \leq\left(x_{1, n-1}-\beta d_{n-1}\right)^{+}$or $x_{1, n} \leq \min \left\{\bar{u}_{1, \infty}\left(x_{2, n-1}\right), \bar{u}_{1, \infty}^{*}\right\}$, and $x_{2, n} \leq\left(x_{2, n-1}-(1-\beta) d_{n-1}\right)^{+}$or $x_{2, n} \leq \min \left\{\bar{u}_{2, \infty}\left(x_{1, n-1}\right), \bar{u}_{2, \infty}^{*}\right\}$ (by definition of $u_{i, \infty}^{*}, i=$ $1,2)$, which then implies that $x_{1, n} \leq \bar{u}_{1, \infty}^{*}$ and $x_{2, n} \leq \bar{u}_{2, \infty}^{*}$ for all $n$ large.

The above theorem tells us that even though the optimal order-up-to level for each type of product depends on system parameters and the initial inventory level of the two products (as given in Theorem 4), when time is sufficiently long, the optimal order-up-to level for each type of product becomes a constant.

Furthermore, in the case when parameters for the two products are the same, such as, $\tau_{1}=\tau_{2}$, etc, the following is true:

Proposition 6 Assume $\bar{u}_{i, \infty}^{*}, i=1,2$, are finite. Suppose $\sigma_{1}=\sigma_{2}, s_{1}=s_{2}, \tau_{1}=\tau_{2}$, then we have

$$
\frac{\bar{u}_{1, \infty}^{*}}{\bar{u}_{2, \infty}^{*}}=\frac{\beta}{1-\beta} .
$$

The proof of Proposition 6 is omitted, and is left to the reader.

By Theorem 5 and Proposition 6, when parameters of the inventory system for the two types of product are the same, we conclude that after sufficient time has elapsed, there is no uninstalling and reinstalling of softwares after demand realization in a period, since it is not necessary as both types of product are either both in excess or in shortage. When parameters of the inventory system for the two types of product are different, then Proposition 6 no longer holds, and in the long run, even though the order-up-to level for each type of product becomes a constant (by Theorem 5), uninstalling and reinstalling of softwares can still occur after demand realization in a period. We also observe that uninstalling and reinstalling of softwares, if occur, will only happen in "one direction" in the long run given that $\beta$ is fixed and that the order-up-to level for each type of product is a constant. Which "direction" this will happen depends on $\beta$ as well as system parameters.

\section{Acknowledgement}

This research is supported by an Agency for Science Technology and Research grant [Grant 1122904020, R314-000-091-305]. The second author would like to thank Prof. Rockafellar for introducing results in Rockafellar and Wets [15] to him. Finally, we would like to thank 
the area editor, Prof. Seshadri, for handling the paper, the associate editor and anonymous reviewer for providing valuable suggestions to improve the paper.

\section{References}

[1] V. Agrawal, X. Chao and S. Seshadri, Dynamic balancing of inventory in supply chains, European Journal of Operational Research, 159(2004), pp. 296-317.

[2] D. P. Bertsekas, Abstract Dynamic Programming, Athena Scientific, Belmont, Massachusetts, 2013.

[3] D. P. Bertsekas, Dynamic Programming and Optimal Control, Volume Two, Athena Scientific, Belmont, Massachusetts, 1995.

[4] S. Chen, The infinite horizon periodic review problem with setup costs and capacity constraints: A partial characterization of the optimal policy, Operations Research, 52(2004), pp. 409-421.

[5] X. Chen, and D. Simchi-Levi, Coordinating inventory control and pricing strategies with random demand and fixed ordering cost: The infinite horizon case, Mathematics of Operations Research, 29(2004), pp. 698-723.

[6] M. Chou, C.-K. Sim, and X.-M. Yuan, Optimal policies for inventory systems with two types of products sharing common hardware platforms: Single period and finite horizon, European Journal of Operational Research, 224(2013), pp. 283-292.

[7] Y. Feng, and Y. Chen, A computational approach for optimal joint inventory-pricing control in an infinite-horizon periodic-review system, Operations Research, 59(2011), pp. 1297-1303.

[8] E. A. Feinberg, Optimality conditions for inventory control, Preprint, 2016.

[9] D. P. Heyman, and M. J. Sobel, Stochastic Models in Operations Research, Volume II, Stochastic Optimization, Dover Publications, Inc., Mineola, New York, 2004.

[10] X. Hu, I. Duenyas, and R. Kapuscinski, Optimal joint inventory and transshipment control under uncertain capacity, Operations Research, 56(2008), pp. 881-897. 
[11] D. Iglehart, Optimality of $(s, S)$ policies in the infinite-horizon dynamic inventory problem, Management Science, 9(1963a), pp. 259-267.

[12] D. Iglehart, Dynamic programming and the analysis of inventory problems, in Scarf, Gilford, Shelly (eds.), Multistage Inventory Models and Techniques, Stanford University Press, Stanford, CA, Chapter 1, 1963b.

[13] E. Ignall, and A. F. Veinott, Jr., Optimality of myopic inventory policies for several substitute products, Management Science, 15(1969), pp. 284 - 304.

[14] C. Paterson, G. Kiesmüller, R. Teunter, and K. Glazebrook, Inventory models with lateral transshipments: A review, European Journal of Operational Research, 210(2011), pp. 125-136.

[15] R. T. Rockafellar, and R. J-B. Wets, Variational Analysis, Springer-Verlag, Berlin Heidelberg, 2004.

[16] G. Tagaras, Effects of pooling on the optimization and service levels of two-location inventory systems, IIE Transactions, 21(1989), pp. 250 - 257.

[17] G. van Ryzin, and G. Vulcano, Optimal auctioning and ordering in an infinite horizon inventory-pricing system, Operations Research, 52(2004), pp. 346-367.

[18] A. F. Veinott, On the optimality of $(s, S)$ inventory policies: New conditions and a new proof, SIAM Journal on Applied Mathematics, 14(1966), pp. 1067-1083.

[19] J. Yang, and Z. Qin, Capacitated production control with virtual lateral transshipments, Operations Research, 55(2007), pp. 1104-1119.

[20] X.-M. Yuan, and K. L. Cheung, Optimal inventory policy for systems with demand cancellations, IIE Transactions, 35(2003), pp. 739-747.

[21] Y. S. Zheng, A simple proof for optimality of $(s, S)$ policies in infinite-horizon inventory systems, Journal of Applied Probability, 28(1991), pp. 802-810. 